\title{
Programa de pós-graduação stricto sensu em terapia ocupacional: fortalecimento e expansão da produção de conhecimento na área
}

\section{Stricto sensu postgraduate program in occupational therapy: strengthening and expanding production of knowledge in the field}

\author{
Ana Paula Serrata Malfitano ${ }^{1}$ \\ Thelma Simões Matsukura ${ }^{1}$ \\ Cláudia Maria Simões Martinez ${ }^{1}$ \\ Maria Luisa Guillaumon Emmel ${ }^{1}$ \\ Roseli Esquerdo Lopes ${ }^{1}$
}

\begin{abstract}
Resumo
Apresenta-se o Programa de Pós-Graduação em Terapia Ocupacional da Universidade Federal de São Carlos, nível Mestrado Acadêmico, iniciado em 2010, primeiro programa da área no Brasil. A preocupação com o campo da pesquisa na terapia ocupacional, bem como com a produção de conhecimento específico, foram os motivadores para a sua criação, também único na América do Sul. Considerando-se a trajetória dos pesquisadores que compõe o seu núcleo docente, o Programa elegeu como área de concentração os "Processos de Intervenção em Terapia Ocupacional”, na perspectiva de que as investigações viessem a contemplar a base epistemológica do campo e as práticas profissionais desenvolvidas pela terapia ocupacional. Duas são suas Linhas de Pesquisa: "Promoção do Desenvolvimento Humano em Contextos da Vida Diária" e "Redes Sociais e Vulnerabilidade". Aponta-se a relevância do Programa no país, discorrendo-se sobre sua caracterização e sobre os desafios para a pesquisa e para a pós-graduação em Terapia Ocupacional.
\end{abstract}

\section{Palavras-chave}

Terapia Ocupacional; Pesquisa; Educação pós-graduada.

\begin{abstract}
It presents the Occupational Therapy Graduate Program at "Universidade Federal de São Carlos" (São Carlos Federal University), academic master level, which started in 2010, first specific program in Brazil. The attention with the research field in occupational therapy, as well as the production of specific knowledge, were the motivators for the creation of this program, unique in South America. The historical of professors resulted in the program's area of concentration "Assistance Processes in Occupational Therapy", with the perspective that the investigations contemplate the epistemological basis of the field and the intrinsic aspects of the practices developed by occupational therapy. There are two Research Areas "Promotion of Human Development in Contexts of Daily Life" and "Social Networks and Vulnerability". We talk about the importance of the program in the country, its characterization and challenges for the field of research and graduate in Occupational Therapy.
\end{abstract}

\section{Keywords}

Occupational Therapy; Research; Postgraduate Education. 


\section{PPGTO-UFSCar: Apresentação}

A preocupação com a pesquisa na terapia ocupacional reflete seu crescimento e desenvolvimento profissionais. $\mathrm{O}$ aumento dos cursos de graduação em universidades públicas no Brasil, a inserção de docentes terapeutas ocupacionais em programas de pós-graduação, bem como a produção de conhecimento no campo da terapia ocupacional vêm se desdobrando em discussões da área acerca de pesquisa, acesso a financiamentos, estabelecimento no sistema brasileiro de pós-graduação e publicação e divulgação acadêmicas.

O aumento de cursos de graduação em terapia ocupacional (desde meados da década de 1970) gerou novas necessidades no mercado de trabalho e a consequente demanda pela formação de profissionais para a docência, colocando em tela a necessidade de produção de conhecimento específico entre os terapeutas ocupacionais. Foi então que estes ampliaram a busca pela formação pós-graduada, restrita inicialmente a Especializações e Aprimoramentos e, mais tarde, dirigida a programas de Mestrado, Doutorado e estágios Pós-Doutorais, delineando o quadro atual.

Nesse cenário, insere-se o Programa de Pós-Graduação em Terapia Ocupacional da Universidade Federal de São Carlos (PPGTO/UFSCar), aprovado pela CAPES (Coordenação de Aperfeiçoamento de Pessoal de Nível Superior), compondo a Área de Educação Física, Fonoaudiologia, Fisioterapia e Terapia Ocupacional (também conhecida como Área 21 da CAPES), o qual iniciou suas atividades em 2010, no nível de mestrado acadêmico, classificado com nota três e que, em breve, passará pela primeira avaliação trienal da CAPES.

O PPGTO da UFSCar organiza-se a partir da Área de Concentração denominada PROCESSOS DE INTERVENÇÃO EM TERAPIA OCUPACIONAL, ou seja, pretende-se que suas investigações contemplem uma perspectiva epistemológica do campo, assim como aspectos intrínsecos das práticas desenvolvidas pela terapia ocupacional. Trata-se de pesquisas sobre intervenções terapêutico-ocupacionais, da avaliação à intervenção, que se debrucem sobre os processos de inclusão e exclusão social, desde a promoção/prevenção até a reabilitação, a que estão submetidas as populações alvo das ações da terapia ocupacional no Brasil.

Como decorrência dessa opção, emergiram suas atuais Linhas de Pesquisa: "Promoção do Desenvolvimento Humano em Contextos da Vida Diária" " "Redes Sociais e Vulnerabilidade”, uma vez que traduzem a vocação e a trajetória histórico -acadêmica dos pesquisadores do Departamento de Terapia Ocupacional (DTO) da UFSCar, os quais, com a colaboração de outros colegas, oferecem sustentação acadêmica ao PPGTO, de maneira coerente e orgânica, em termos de suas produções, projetos e orientações.

Centram-se nessas linhas os estudos relacionados com o desenvolvimento típico e atípico do ser humano; a análise e a adaptação de atividades do cotidiano para populações com deficiências, com incapacidades temporárias ou permanentes; o desenvolvimento de novas tecnologias de intervenções terapêutico-ocupacionais que minimizem a dependência e facilitem a autonomia nos diversos contextos da vida diária (em casa, na escola, no trabalho, na vida social); a inclusão de populações em processos de ruptura das redes sociais de suporte; o estudo de políticas públicas voltadas para os grupos populacionais aos quais se voltam as ações da terapia ocupacional; as pesquisas sobre a produção e aplicação de novos conhecimentos e tecnologias de intervenção em terapia ocupacional para a abordagem de problemas sociais. 
Essas temáticas constituem objetos de investigação em terapia ocupacional, socialmente relevantes, que respondem a muitas necessidades da sociedade brasileira.

\section{Linha 1 - Promoção do Desenvolvimento Humano nos Contextos da Vida Diária}

Tem por objetivo o estudo das intervenções em terapia ocupacional, nos contextos da vida diária das pessoas ao longo do seu ciclo de vida, sob a ótica do desenvolvimento humano, contemplando processos de funcionalidade e de incapacidade, bem como as situações de risco.

Seus fundamentos epistemológicos apoiam-se no estudo de bases neurobiológicas, cognitivas, psicossociais do desenvolvimento e dos processos de atenção em saúde para respaldar investigações que buscam compreender como a atividade humana e o ambiente interferem na promoção do desenvolvimento saudável, do desempenho funcional, da independência e da autonomia do ser humano, dentro de uma perspectiva voltada para a aquisição de habilidades e de oportunidades de inserção social.

Lança mão de métodos descritivos e experimentais de investigação. Seus estudos visam à criação de tecnologias sociais, de recursos terapêuticos e de intervenções que facilitem e estimulem o desenvolvimento, o desempenho funcional e o engajamento em ocupações significativas nos diversos contextos cotidianos, nas situações de limitações, temporárias ou permanentes, objetivando promover melhoria da qualidade de vida.

\section{Linha 2 - Redes Sociais e Vulnerabilidade}

Objetiva estudar as intervenções de terapia ocupacional com as populações em situação de vulnerabilidade social e o desenvolvimento de tecnologias sociais de inserção, participação e autonomia. Dialoga com temáticas sociais, tais como a pobreza, as políticas sociais, a ocupação do espaço urbano, a identidade cultural, o acesso a serviços sociais (saúde, educação, cultura, assistência social, justiça e outros) e correlatas.

São desenvolvidas pesquisas com foco nas crianças e adolescentes em situação de vulnerabilidade social, na juventude contemporânea, nas populações em situação de rua, na inserção e participação social de pessoas com deficiência e de pessoas com transtorno mental, na geração de renda e de valor com relação ao mundo do trabalho.

Seu aporte epistemológico vem da Saúde Coletiva, da Educação, das Ciências Sociais, especialmente, da Antropologia e da Sociologia, e da Terapia Ocupacional Social; lidando com a fundamentação de estudos acerca das práticas e dos processos de transformação cultural e de exclusão/inclusão social, da atenção territorial e comunitária, da educação, formal e não formal, combinando referências para a construção das metodologias de ação requeridas nesses contextos.

\section{Corpo Docente}

Em 2013, o PPGTO-UFSCar conta com um corpo docente formado por 14 pesquisadores, sendo 11 do seu núcleo permanente e 3 colaboradores.

A Linha de Pesquisa "Promoção do Desenvolvimento Humano em Contextos da Vida Diária” aglutina os seguintes docentes: Cláudia Maria Simões Martinez, 
Daniel Marinho Cezar da Cruz, Iracema Serrat Vergotti Ferrigno, Maria Luísa Guillaumon Emmel e Thelma Simões Matsukura. Todos docentes pesquisadores do Departamento de Terapia Ocupacional (DTO) da UFSCar. Conta ainda com a colaboração de Lívia de Castro Magalhães e Marisa Cotta Mancini, da área de terapia ocupacional da Universidade Federal de Minas Gerais, e de Marina Silveira Palhares, docente aposentada do DTO/UFSCar.

A Linha de Pesquisa "Redes Sociais e Vulnerabilidade" é formada pelas docentes: Ana Paula Serrata Malfitano, Isabela Aparecida de Oliveira Lussi e Roseli Esquerdo Lopes, do DTO/UFSCar, Marisa Bittar, do Departamento de Educação da UFSCar e, ainda, por Denise Dias Barros e Fátima Correa Oliver, da área de terapia ocupacional da Universidade de São Paulo (USP), todas compondo o núcleo permanente de docentes.

\section{Processos Seletivos e o funcionamento do PPGTO- UFSCar}

O PPGTO-UFSCar realiza processos seletivos anuais, no segundo semestre de cada ano, tendo apresentado uma demanda crescente desde a sua primeira edição, com a presença de candidatos de várias regiões do Brasil e de outros países.

Direciona-se a graduados em terapia ocupacional e em outros cursos de interface (como enfermagem, fisioterapia, educação física, psicologia, pedagogia, ciências sociais, educação especial), com o intuito de formar profissionais da saúde, da educação e do campo social que desenvolvam conhecimento e tecnologias voltadas às temáticas dos contextos do desenvolvimento, dos cotidianos e dos processos de inserção social.

Todas as informações estão disponíveis em: www.ppgto.ufscar.br

\section{PPGTO-UFSCar: objetivos e desenvolvimento}

O Programa de Pós-Graduação em Terapia Ocupacional da UFSCar, iniciando com o Mestrado Acadêmico, foi elaborado considerando-se o princípio que um programa de pós-graduação tem por função a produção de novos pesquisadores, novos conhecimentos, sua disseminação e sua utilização, funcionando como um centro multiplicador de ideias e contribuindo para o crescimento de uma determinada área de conhecimento. Trata-se de consolidar e ampliar conhecimentos já adquiridos, como também de se projetar em direção às novas tendências na área, tendo como objetivo específico a formação de Mestres em Terapia Ocupacional capacitados a explorarem as ferramentas que constituem as bases da terapia ocupacional, focadas, no nosso caso, nas temáticas do Desenvolvimento Humano e da Inserção Social.

Assim, os objetivos principais deste primeiro PPGTO, stricto sensu, são fruto de demandas presentes no território nacional e latino-americano relacionadas ao desenvolvimento científico do campo e à necessidade de formação pós-graduada em terapia ocupacional.

O PPGTO/UFSCar visa, igualmente, à formação de recursos humanos mais bem capacitados para atuarem no ensino superior, em franca expansão no território brasileiro.

Vale esclarecer que a formação de docentes terapeutas ocupacionais é, ainda, incipiente num contexto de ampliação exponencial de cursos de graduação.

Em termos gerais, o Brasil conta com 78 cursos de graduação (segundo o Instituto Nacional de Estudos e Pesquisas Educacionais Anísio Teixeira - INEP, dados 
de julho de $2010^{1}$. Todos estão autorizados pelo INEP/MEC, porém nem todos estão efetivamente em funcionamento) havendo, portanto, uma demanda de formação docente na área. Se tomarmos como média 15 docentes para a área específica dos cursos de graduação em terapia ocupacional (conforme parâmetros atuais do Ministério da Educação do Brasil), podemos nos reportar a uma demanda de mais de 1.100 docentes para aqueles 78 cursos. De acordo com levantamento da RENETO (Rede Nacional de Ensino e Pesquisa em Terapia Ocupacional, fundada como uma rede virtual, sob uma coordenação colegiada, em 2000, e formalizada juridicamente como uma entidade de âmbito nacional de caráter associativo e educacional em 2005, congregando associados individuais e institucionais) ${ }^{2}$, projetava-se para o final de 2011, no Brasil, a existência de apenas cerca de 130 doutores com graduação na terapia ocupacional. Fica, então, explícita a demanda referida.

Desse modo, avalia-se que o PPGTO viabilizará tanto a formação de um contingente represado de terapeutas ocupacionais quanto a produção de conhecimento específico.

Não há dúvida de que a pós-graduação stricto sensu é a grande impulsionadora da produção científica de uma área, uma vez que no Brasil, como em boa parte do mundo, se começa a falar de pesquisa específica quando se fala de Mestrado e Doutorado dedicados às temáticas de um campo; esse é o lócus em que a oportunidade se instala, onde se articulam espaço e tempo para a experimentação, para o estudo, para a pesquisa e, consequentemente, para a reflexão.

\section{A produção de conhecimento em Terapia Ocupacional: desafios para a área}

Analisando as questões próprias da área, ainda que inseridas em um contexto mais amplo, considera-se fundamental o desenvolvimento de conhecimento científico específico acerca da contribuição da intervenção terapêutico-ocupacional por meio das políticas nacionais de saúde, de assistência social, de cultura e de educação, particularmente no tocante às proposições inclusivas para grupos com relação aos quais dedicamos nossa atenção.

Acredita-se que o crescimento e o fortalecimento da área no nível da pesquisa e da pós-graduação stricto sensu contribuem para a compreensão e a intervenção em problemáticas contemporâneas, como também para o desenvolvimento de estratégias em prol das populações atendidas pelo terapeuta ocupacional, tais como pessoas em sofrimento psíquico e/ou com deficiências e incapacidades, excluídos sociocultural e economicamente da vida comum, entre outros.

Assim, debate-se sobre "como articular, na terapia ocupacional brasileira, a correlação entre o desenvolvimento da área, ou seja, seu esforço em ciência, tecnologia e inovação, e os processos de inclusão-exclusão social a que estão submetidas as populações alvo de suas intervenções profissionais" no Brasil ${ }^{3}$. Tais questionamentos avançarão na medida em que possamos ganhar espaços institucionais e legitimidade acadêmico-científica.

Frente ao atual quadro de institucionalidade da pesquisa e da pós-graduação no cenário brasileiro, por meio de um levantamento dos Grupos de Pesquisa cadastrados no Diretório de Grupos de Pesquisa do Conselho Nacional de Desenvolvimento Científico e Tecnológico - CNPq, na grande área da Saúde, onde se insere a subárea de Fisioterapia e Terapia Ocupacional, que apresentassem o termo "Terapia Ocupacional" no nome do grupo, no título de linha de pesquisa ou em suas palavras-chave; encontraram-se 32 grupos registrados ${ }^{4}$, demonstrando um crescimento 
significativo desde $2008^{5}$. Entretanto, este panorama não se refletiu, ainda, em solicitações e aumento de demandas aos órgãos responsáveis pela pesquisa no país.

Com relação à atuação na pós-graduação, até 2009, os docentes estavam vinculados a programas de áreas correlatas, pois não havia nenhum programa específico em terapia ocupacional no Brasil ${ }^{6}$. Na grande área de Saúde, subárea Educação Física, Fonoaudiologia, Fisioterapia e Terapia Ocupacional (Área 21) da CAPES, há três programas reconhecidos que contam com a participação de pesquisadores da terapia ocupacional, sendo eles: o Programa em Ciências da Reabilitação da Universidade Federal de Minas Gerais (UFMG), o Programa em Ciências da Reabilitação da Universidade de São Paulo (USP) e o Programa de Pós-Graduação em Terapia Ocupacional da Universidade Federal de São Carlos (UFSCar).

O estado da arte da terapia ocupacional no país, assim como em toda a América Latina, é ainda incipiente e concentra grandes desafios para sua ampliação, reconhecimento e institucionalização na própria área e, igualmente, junto aos órgãos acadêmico-científicos de apoio à pesquisa e à pós-graduação stricto sensu.

O processo histórico vivenciado até aqui, que teve o empenho de diferentes gerações de pesquisadores terapeutas ocupacionais, reflete esforços e avanços coletivos para o crescimento acadêmico-institucional da terapia ocupacional. Porém, são muitos os desafios para a manutenção da pós-graduação stricto sensu, como também é urgente lidarmos com a necessidade de sua ampliação, interna e externa à UFSCar, para que haja condições de fortalecimento e expansão da produção de conhecimento próprio.

Os diálogos e inserções da terapia ocupacional na esfera da pesquisa não estão institucionalizados e envolvem disputas em campos já consolidados e em debate acerca de suas regras, funcionamento e acessos.

A concreta participação da área dependerá da capacidade de organização coletiva para ocupação dos espaços legitimados na CAPES, no CNPq e nos demais órgãos de fomento à pesquisa e à pós-graduação no país; igualmente, dependerá da presença da terapia ocupacional no desenvolvimento de pesquisas e conhecimentos que sejam relevantes para a área e se aglutinem no ponto comum de trabalho: a promoção da autonomia e a inclusão de sujeitos, individuais e coletivos, com os quais trabalhamos.

Está posta para a terapia ocupacional a possibilidade de crescimento também no campo da pesquisa, sendo que, para tanto, é necessário que enfrentemos os processos de qualificação de seus pesquisadores, de aumento de doutores, de criação de novos programas stricto sensu, de qualificação e reconhecimento dos seus periódicos específicos, de acesso ao financiamento de pesquisas e, sobretudo, de tensionamento para que haja a valorização das análises de práticas sociais dedicadas à compreensão e abordagem das temáticas que interpelam a área ${ }^{7}$.

\section{Contribuição das autoras}

Todas as autoras compuseram a Comissão que elaborou a proposta do Programa de Pós-Graduação em Terapia Ocupacional da UFSCar, apresentada à CAPES em 2009, e estão diretamente envolvidas com sua implementação e consolidação. Todas as autoras são responsáveis pela redação e revisão do manuscrito.

\section{REFERÊNCIAS}

1. http://portal.inep.gov.br/. Acesso em 25 de julho de 2010.

2. www.reneto.org.br. Acesso em 11 de fevereiro de 2013. 
3. Lopes RE, Malfitano APS, Oliver FC. XI Encontro Nacional de Docentes de Terapia Ocupacional: refletindo sobre os processos de formação acadêmica e profissional. Rev Ter Ocup Univ São Paulo 2008; 19:159-166.

4. http://dgp.cnpq.br/buscaoperacional/. Acesso em 11 de fevereiro de 2013.

5. Lopes RE, Malfitano, APS, Oliver, FC, Sfair SC,Medeiros TJ. Pesquisa em terapia ocupacional: apontamentos acerca dos caminhos acadêmicos no cenário nacional. Rev Ter Ocup Univ São Paulo 2010; 21:207-214.

6. Oliver FC. Disseminação do conhecimento e periódicos científicos: contribuições para o debate em terapia ocupacional. Cad Ter Ocup UFSCar 2009; 17:69-85.

7. Lopes RE, Malfitano APS. Perspectivas e desafios para a pesquisa em terapia ocupacional: uma análise do seu I Seminário Nacional de Pesquisa. Cad Ter Ocup da UFSCar 2009; 17:115-120.

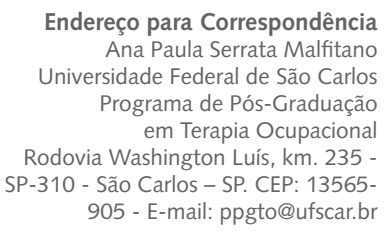

$$
\begin{array}{ll}
\text { Recebido } & 19 / 03 / 2013 \\
\text { Revisado } & 19 / 03 / 2013 \\
& 25 / 03 / 2013 \\
& 31 / 03 / 2013 \\
\text { Aprovado } & 02 / 04 / 2013
\end{array}
$$

